\title{
A Millimeter-Wave Instrument for Use in a Synergistic, Optical/Millimeter-Wave Approach to Pollution Monitoring
}

\author{
J. Bredow \\ D. Gibbs \\ A.K. Fung \\ S. Tjuatja \\ P. Girardot \\ University of Texas at Arlington \\ PO Box 19016 \\ Arlington, TX 76019 \\ Phone: (817)-273-3497 \\ FAX: (817)-273-2253
}

\begin{abstract}
A multisensor approach is being developed at the University of Texas at Arlington (UTA) to improve upon current capabilities in environmental monitoring. These sensors consist of an FT-IR for operation in the near to thermal infrared regions of the spectrum, a long-path UV (LPUV) system and an active/passive millimeter-wave instrument that will operate over the $75-110 \mathrm{GHz}$ window. This report will discuss how we expect the synergistic capability to work and will describe in some detail the design of the millimeter-wave instrument. Field-portable FT-IR instruments have been used effectively to monitor a limited number of gaseous pollutants but are limited by interference from $\mathrm{CO}_{2}$ and $\mathrm{H}_{2} \mathrm{O}$ and, for longer hydrocarbon chains, by spectral overlap. UV instruments, on the other hand, are much more sensitive but many gases do not exhibit spectral absorption (emission) in the UV. It is known that various hydrocarbons, $\mathrm{NO}_{\mathrm{x}}, \mathrm{O}_{3}$ and other gases have rotational lines in the $75-110 \mathrm{GHz}$ region, and narrow-bandwidth millimeter-wave instruments are now being effectively utilized to monitor trace gases in the stratosphere. Use of a wide-bandwidth millimeter-wave system is now being proposed for environmental monitoring at lower altitudes.

Aspects of the millimeter-wave system that are considered in this report include antenna and front-end mixer selection, active/passive mode selections and parallel/serial channel tradeoffs. For this instrument $1200^{\circ} \mathrm{K}$ (cooled) effective noise is expected.
\end{abstract}

\section{Introduction}

Recently passed "clean air" legislation has made wide-area pollution monitoring capabilities essential, both for the targeted industries (to ensure that they are within compliance) and for those responsible for enforcing the legislation. Presently, standardized methods for monitoring pollutants are based upon either the traditional point monitors -which determine the concentration of a single pollutant at a point -- or extractive sampling (extraction of an air sample for subsequent laboratory analysis). Both methods have severe limitations: (1) they are not cost effective as an enormous array of monitors/samplers is needed to characterize extended-area plumes; (2) collection of a large number of samples is required to analyze a contaminant plume with any degree of certainty due to the turbulent wind carrying the plume; (3) these methods almost never provide high-quality, legally admissible air data due to the limitations associated with point monitoring techniques; and (4) during extractive sampling, sample adsorption by the canister wall has been shown to be a significant loss mechanism. Remote sensing instruments are attractive as monitoring systems for several reasons: (1) they do not require collection of air samples; (2) they can probe "hard-to-reach" regions such as plumes from smoke stacks and hazardous landfills; and (3) they are cost-effective as a result of their near real-time, wide-area monitoring of path-averaged concentrations of multiple pollutants.

Remote sensing instruments that operate over a single band of the spectrum, i.e., IR, UV or millimeter-waves, are incapable of completely satisfying the monitoring needs addressed by the new legislation. Hence, the Wave Scattering Research Center (WSRC) at UTA is investigating the synergistic use of IR, UV and millimeter-waves for 
identifying and quantifying pollutants. The IR region contains absorption lines of many compounds of interest, but also is noisy due to interference from background gases (i.e., $\mathrm{O}_{2}$ and $\mathrm{H}_{2} \mathrm{O}$ ). UV is relatively quiet but only a small number of compounds of interest absorb in this region. The millimeter-wave region of the spectrum, the topic of this report, contains information about pollutants that is not available in the IR and UV regions. In addition, millimeter-waves are not affected by particulates, fog and haze, to the extent that the IR and UV regions are.

We anticipate that the three instruments will be used primarily in the absorption mode, where a signal is transmitted by each instrument through the polluted air is intercepted by a retroreflector and returned to the instrument receiver. The combined IR, UV and millimeter-wave absorption dataset is then processed simultaneously to identify and quantify individual pollutants according to their spectral "fingerprint."

\section{Description of the $75-110 \mathrm{GHz}$ pollution monitoring instrument}

Figure 1 indicates the block diagram of the $75-110 \mathrm{GHz}$ active/passive pollution monitoring instrument. There are four basic components to this system that will be dealt with separately: (1) the transmitter; (2) the quasi-optical section at the input to the receiver; (3) the heterodyning section of the receiver; and (4) the signal processing section of the receiver. In the following discussion, keep in mind that the instrument can be used to measure absorption thorough a polluted region; emission from a region where hot gases are present; or, to a limited extent, to measure scattering from particulates in a plume.

\subsection{Transmitter}

In the absorption mode, for ranges up to a few hundred meters, the transmitted signal will consist of spectral lines at $100 \mathrm{MHz}$ spacing over the entire $75-110 \mathrm{GHz}$ region. This is accomplished by means of a comb generator that generates impulses in synchronization with a $100 \mathrm{MHz}$ signal, yielding frequency components spaced by $100 \mathrm{MHz}$ over 100 $\mathrm{MHz}$ to $18 \mathrm{GHz}$. These are then upconverted in a double-sideband mode to cause $100 \mathrm{MHz}$-spaced frequency components to occur over the entire 75$110 \mathrm{GHz}$ region. In the absorption mode, for ranges exceeding a few hundred meters, we will reduce the bandwidth covered instantaneously so as to achieve an acceptable $\mathrm{S} / \mathrm{N}$ at the receiver. The pollutant information will then be obtained by examining the absorption pattern as a function of frequency. In the scattering mode, the $93 \mathrm{GHz}$ PLO, with output power of about $30 \mathrm{~mW}$, will be pulse modulated. Notice that the switching needed to achieve this is not shown in the figure.

\subsection{Antennas and quasi-optical section at input to receiver}

In order to keep the receiver and transmitter gains as high as possible, without excessive cost, we are making use of cassegrain-fed parabolic dish antennas. Note that high gain is needed in order to make the best possible use of the low millimeterwave power that is available. With $45-50 \mathrm{~dB}$ gain for both transmitter and receiver a few microwatts of power can be useful up to a few hundred meters.

At the output of the receive antenna a scalar horn is used to generate a gaussian pattern so that we can take advantage of the low loss and flexibility possible with quasi-optical processing. Two dichroic plate filters are used to divide the bands into two receiver channels; the primary reason for two channels is to keep the noise temperature reasonably low in each channel by keeping the IF bandwidth less than about $10 \mathrm{GHz}$. The configuration that is shown also ensures that the input to the heterodyning mixer is matched at the image frequency, meaning that the best possible noise temperature is obtained.

The reader should notice that the optical configuration as shown will permit coverage of only part of the $75-110 \mathrm{GHz}$ band - a slight reconfiguration of the optics will be done to cover the remaining segment. The $75-110 \mathrm{GHz}$ band is covered in four segments, $75-84 \mathrm{GHz}, 84-93 \mathrm{GHz}$, 93-102 GHz and $102-110 \mathrm{GHz}$. With a fixed IF for both channels of 4.5 to $13.5 \mathrm{GHz}$ the band can be covered by appropriately selecting the upper or lower sidebands for the two channels. For example, one mixer is supplied by an $88.5 \mathrm{GHz}$ local oscillator so that the upper sideband at RF is $93-102 \mathrm{GHz}$, while the lower sideband is $75-84 \mathrm{GHz}$. In a similar fashion the mixer supplied by the $97.5 \mathrm{GHz}$ local oscillator covers the two remaining segments of the 75-110 GHz band.

When operated in the passive, i.e., radiometer, mode a hot and cold calibration load are inserted, by means of a chopper wheel, into the signal path at the location shown in the figure. 
2.3 Heterodyning section of the receiver

To facilitate processing data from the 75 $110 \mathrm{GHz}$ region, the signal is heterodyned in the 4.5 to $13.5 \mathrm{GHz}$ region of the spectrum (as per discussion above). To ensure that the heterodyning is done coherently with respect to the transmitted signal, the $88.5 \mathrm{GHz}$ and $97.5 \mathrm{GHz}$ local oscillators are phase-locked to the $93 \mathrm{GHz}$ phase-locked oscillator of the transmitter. This is of particular importance for the absorption mode where a very narrow bandwidth is needed to process each of the $100 \mathrm{MHz}$-spaced lines for the purpose of obtaining the highest possible $\mathrm{S} / \mathrm{N}$.

One final note: for the radiometer mode the heterodyning mixers and IF amplifiers can be cooled to $77^{\circ} \mathrm{K}$; this allows us to reduce the noise temperature of the receiver.

\subsection{Signal processing section of the receiver}

The signal processing section will consist of parallel and serial components. In the absorption mode with $100 \mathrm{MHz}$ spacing between spectral lines, 360 outputs are needed to characterize absorption over the entire $75-110 \mathrm{GHz}$ region. Although this section of the instrument is still being designed, we anticipate that 20 to 30 parallel channels will be used. As a result 12 to 18 time segments will be required to obtain outputs from all 360 spectral lines.

\subsection{Summary}

A combined IR, UV and millimeter-wave approach to pollution monitoring is being developed at WSRC; this report has indicated the fundamental impetus behind this development. The basic design features of the millimeter-wave instrument, now under development, have also been discussed.

\section{Acknowledgment}

Funding for components of the millimeterwave system is being provided by the National Science Foundation.

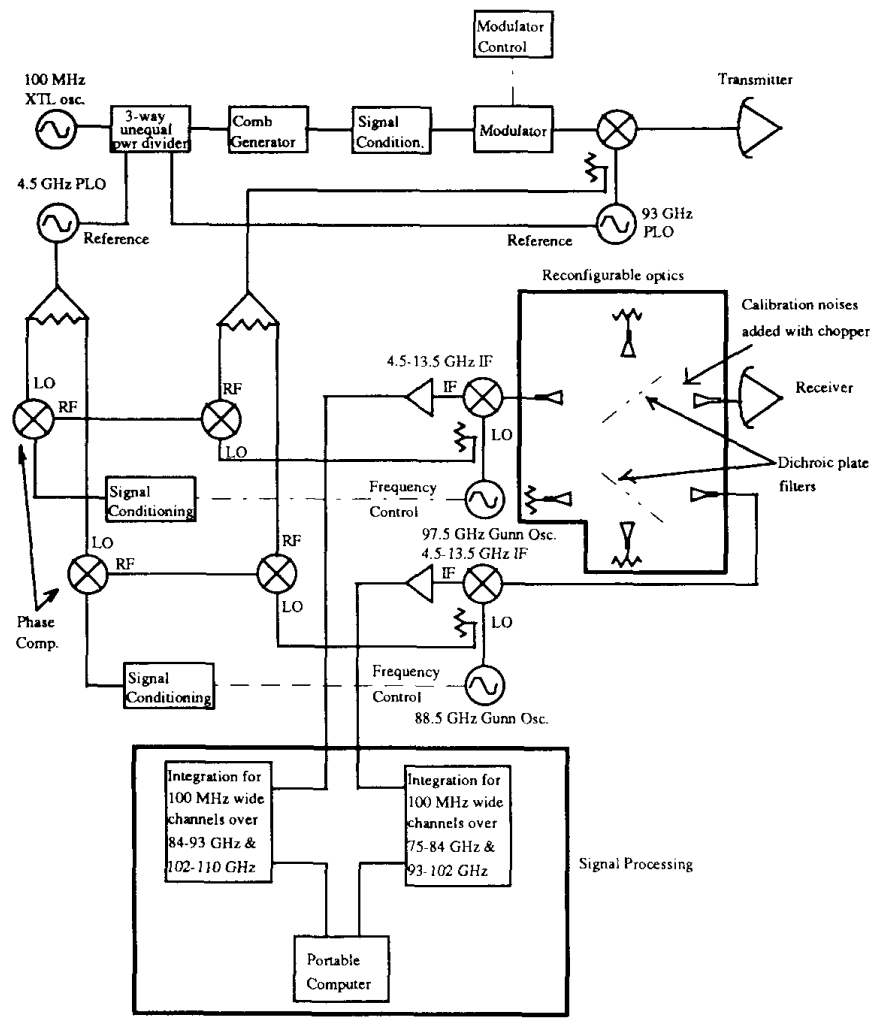

Figure 1: Block diagram of the $75-110 \mathrm{GHz}$ pollution monitoring instrument. 